\title{
Implantation of fetal rat lung fragments into bleomycin-induced pulmonary fibrosis
}

\author{
Hiroaki Toba, MD, ${ }^{\text {a }}$ Shoji Sakiyama, $\mathrm{MD}, \mathrm{PhD},{ }^{\mathrm{a}}$ Koichiro Kenzaki, MD, PhD, ${ }^{\mathrm{a}}$ Yukikiyo Kawakami, $\mathrm{MD},{ }^{\mathrm{a}}$ \\ Koh Uyama, MD, ${ }^{a}$ Yoshimi Bando, $\mathrm{MD}, \mathrm{PhD},{ }^{\mathrm{b}}$ and Akira Tangoku, $\mathrm{MD}, \mathrm{PhD}^{\mathrm{a}}$
}

\begin{abstract}
Objective: Pulmonary fibrosis is a life-threatening disease that results in progressive respiratory failure. We have suggested the possibility of fetal lung tissue as an option for further investigation into lung regeneration. The objective was to prove whether fetal lung fragments can survive and differentiate in fibrotic lung.

Methods: Lewis rats were administered bleomycin and used as recipients after 3 or 4 weeks. Day 17 fetal lung tissue from green fluorescent protein Lewis rats was used as donor material. Donor lungs were removed, cut into small pieces, and implanted into the recipients' left lung. The recipients received cyclosporin to prevent immune response to green fluorescent protein and were killed after 1, 2, 4, 8, and 12 weeks and histologically evaluated. Furthermore, the expression of thyroid transcription factor- 1 and Clara cell secretory protein in the implanted fetal lung tissue was immunohistologically evaluated.

Results: Fibrotic changes were recognized for a long period of time in the recipient lungs. The implanted fetal lung fragments could be clearly distinguished from recipient lungs because of the luminescence of grafts. Fetal lung fragments could survive in the recipient lungs with fibrotic changes. The air spaces of implanted fetal lungs were narrow at 1 and 2 weeks but expanded with the passage of time. The connection between the recipient lung and the implanted fetal lung was recognized, particularly in the peripheral grafts. The expression patterns of thyroid transcription factor- 1 and Clara cell secretory protein in implanted lungs resembled those in the process of normal lung morphogenesis.
\end{abstract}

Conclusions: Fetal rat lung fragments could survive and differentiate in bleomycin-induced completely fibrotic lung. (J Thorac Cardiovasc Surg 2012;143:1429-35)

Idiopathic pulmonary fibrosis is a chronic progressive and potentially fatal lung disease characterized by the proliferation of fibroblasts and deposition of extracellular matrix. ${ }^{1,2}$ Kim and colleagues ${ }^{3}$ indicated that its prognosis was poor and that survival time from diagnosis ranged from 2 to 4 years. Although anti-inflammatory drugs such as prednisone and cytotoxic drugs (eg, cyclophosphamide and azathioprine) are used for treatment, they do not halt the progression of fibrosis or clearly improve lung function or life expectancy. ${ }^{1,4}$ To date, although various drugs have been used for clinical trials, none has shown success comparable to that of antifibrotic drugs. ${ }^{4}$ At present, lung transplantation may be considered an appropriate therapy.

\footnotetext{
From the Departments of Thoracic and Endocrine Surgery and Oncology, ${ }^{\mathrm{a}}$ The University of Tokushima, Kuramoto-cho, Tokushima, Japan; and Molecular and Environmental Pathology, ${ }^{\mathrm{b}}$ Institute of Health Biosciences, The University of Tokushima, Kuramoto-cho, Tokushima, Japan

Disclosures: Authors have nothing to disclose with regard to commercial support.

Received for publication Nov 4, 2011; revisions received Dec 13, 2011; accepted for publication Jan 4, 2012; available ahead of print Feb 1, 2012.

Address for reprints: Shoji Sakiyama, MD, PhD, Department of Thoracic and Endocrine Surgery and Oncology, Institute of Health Biosciences, The University of To-

kushima, Kuramoto-cho, Tokushima 770-8503, Japan (E-mail: sakiyama@clin.

med.tokushima-u.ac.jp).

$0022-5223 / \$ 36.00$

Copyright (C) 2012 by The American Association for Thoracic Surgery

doi:10.1016/j.jtcvs.2012.01.002
}

Several potential sources of cells for airway epithelium repair have been identified. These are divided into exogenous stem cells from other tissue in the body and endogenous progenitor cells present in the respiratory tract. ${ }^{5}$ Rojas and colleagues ${ }^{6}$ reported that bone marrow-derived stem cells, which are an example of exogenous stem cells, could integrate into alveolar epithelium and contribute to lung regeneration in a model of bleomycin (BLM)-induced pulmonary fibrosis. However, some studies have failed to restore alveolar epithelium. ${ }^{7,8}$ Furthermore, although stem cells such as embryonic stem cell-induced pluripotent stem cells can also be expected as new sources for lung regeneration, no direct applications to airway cells have been reported. ${ }^{5}$ On the other hand, Serrano-Mollar and colleagues ${ }^{9}$ reported that transplantation of alveolar type II cells, which are an example of endogenous progenitor cells, reversed BLM-induced pulmonary fibrosis in rats. However, this was considered to be a limited method to prevent the fibrotic process. As such, tissue engineering of the lung with various cell sources is still difficult because of problems to select (1) the cell source with the highest potential, (2) the best biocompatible and degradable scaffold suitable for lung development, (3) the best combination of growth factors and culture conditions to promote cellular differentiation, and (4) the best conditions to support 3-dimensional production of lung tissues. ${ }^{10}$ 


\section{Abbreviations and Acronyms \\ BLM = bleomycin \\ CCSP $=$ Clara cell secretory protein \\ CyA $=$ cyclosporin $\mathrm{A}$ \\ GFP = green fluorescent protein \\ $\mathrm{OCT}=$ optimal cutting temperature \\ TTF-1 $=$ thyroid transcription factor- 1}

On the other hand, we have attempted lung regeneration using fetal rat lung tissue as another approach. ${ }^{11}$ Fetal lung tissue has (1) great potential for further growth, (2) excellent potential for differentiation and proliferation, and (3) mesenchymal tissue as a suitable scaffold. ${ }^{12}$ In a previous study, we made use of its characteristics and investigated whether fetal rat lung tissue implanted into adult lung parenchyma could survive, grow further, and differentiate. We could consequently suggest the possibility of fetal lung tissue as an option for further investigation into lung regeneration. ${ }^{11}$ In this study, we made a long-term model of BLM-induced pulmonary fibrosis, implanted fetal rat lung fragments into fibrotic lung, and investigated whether the graft could survive.

\section{MATERIALS AND METHODS \\ Animals}

This study was approved by the Animal Care Committee of the University of Tokushima. Specific pathogen-free male Lewis rats aged approximately 8 to 10 weeks were obtained from Charles River Japan, Inc (Kanagawa, Japan) and used as recipients.

Green fluorescent protein (GFP) Lewis fetal rat lungs were used as donors to clarify the borderline between donor and recipient. GFP Lewis rats were provided by the Division of Organ Replacement Research, Center for Molecular Medicine, Jichi Medical University School of Medicine. $^{13}$

Rats were maintained in an animal house in a pathogen-free environment, with a 12-hour light-dark cycle, and provided free access to water and food. The animals received humane care in compliance with the institutional and national guidelines (Japanese Ministry of Education, Culture, Sports, Science and Technology).

\section{Bleomycin Hydrochloride-Induced Pulmonary Fibrosis}

BLM hydrochloride was purchased from Nippon Kayaku Co (Tokyo, Japan). Intratracheal administration of BLM was performed according to the method described by Goto and colleagues. ${ }^{14}$ Each rat was anesthetized by an intraperitoneal injection of ketamine hydrochloride $(20 \mathrm{mg} /$ $\mathrm{kg}$ ) and diazepam $(7.5 \mathrm{mg} / \mathrm{kg})$, tracheostomy was surgically performed, and a catheter made using a polyethylene tube (internal diameter 0.2 $\mathrm{mm}$, outer diameter $0.5 \mathrm{~mm}$; Natsume Seisakusho Co, Japan) beforehand was inserted between the cartilaginous rings and placed in the trachea. Then, the catheter was subcutaneously tunneled to the nape of the neck. Three days after insertion of the catheter, BLM at a total dose of $4.5 \mathrm{mg} / \mathrm{kg}$ was administered in 4 separate doses $(0.5 \mathrm{~mL} / \mathrm{kg} /$ time $)$ at intervals of 2 hours to the rats via the catheter with $1.5 \%$ halothane $\left(\mathrm{N}_{2} \mathrm{O}: \mathrm{O}_{2}=1: 1\right)$. Three to four weeks after administration of BLM, rats were used as recipients. Control animals received the same volume of saline in the same manner.

\section{Preparation of Donors}

Female Lewis rats aged approximately 8 to 10 weeks were chosen and mated with GFP male Lewis rats for only 1 day. Seventeen days later, pregnant rats were killed. After their fetuses were removed, only GFP fetuses with fluorescence by excitation light (ultraviolet A; $365 \mathrm{~nm}$ ) were selected and used. Both their lungs were dissected under a microscope, cut into fine pieces, and stored in Dulbecco's modified Eagle's medium containing 10\% fetal bovine serum and penicillin-streptomycin (Life Technologies Corp, Carlsbad, Calif). ${ }^{15}$

\section{Implantation Procedure}

BLM-administered rats were used as recipients. They were ventilated with a small animal respirator (SN480-7, Shinano, Tokyo, Japan) at 70 cycles/min, a tidal volume of $10 \mathrm{~mL} / \mathrm{kg}$, and a positive end-expiratory pressure of $2 \mathrm{~cm} \mathrm{H}_{2} \mathrm{O}$ under atropine sulfate at $0.1 \mathrm{mg} /$ body (administered subcutaneously) and $1.5 \%$ halothane $\left(\mathrm{N}_{2} \mathrm{O}: \mathrm{O}_{2}=1: 1\right)$. After left thoracotomy, GFP fetal lung fragments were injected just under the pleura of the left lung apex, at a volume of $0.1 \mathrm{~mL}$ that contained lung fragments from 2 fetuses, using a 0.25 -mL syringe and 20-gauge needle. After implantation, they immediately received $10 \mathrm{mg} / \mathrm{kg}$ cyclosporin A (CyA) (dissolved in olive oil and absolute alcohol and injected intramuscularly) to prevent an immune response to GFP protein. In addition, they received 4 $\mathrm{mg} / \mathrm{kg}$ CyA on the first day after implantation and subsequently $1 \mathrm{mg} / \mathrm{kg}$ every other day until sacrifice. BLM-administered rats that received only CyA by the same regimen were used as vehicle control.

\section{Experimental Groups}

Animals were randomly distributed into 4 groups: (1) saline group $(\mathrm{n}=24)$, saline administration; (2) BLM group $(\mathrm{n}=24)$, BLM administration; (3) implantation group $(\mathrm{n}=20)$, BLM administration plus implantation of GFP fetal rat lung fragment and CyA administration; and (4) control group $(\mathrm{n}=25)$, BLM administration and CyA administration.

In the saline and BLM groups, 6 animals were killed at 4, 8, 12, and 16 weeks. Four animals in the implantation group and 5 animals in the control group were killed at $1,2,4,8$, and 12 weeks.

\section{Histologic Study}

The lungs were perfused with saline through the pulmonary artery after cutting the left atrium and then removed en bloc with the heart. In the implantation group, they were fixed with fixing solution (4\% paraformaldehyde: optimal cutting temperature $[\mathrm{OCT}]$ compound $=4: 1$ ) from the trachea at $15 \mathrm{~cm} \mathrm{H}_{2} \mathrm{O}$. After the specimens were preserved at 4 degrees for 5 hours in a dark room and the position of donors was identified from fluorescence by excitation light (ultraviolet A; $365 \mathrm{~nm}$ ), they were embedded in OCT compound and preserved by freezing. Later, they were cut at $10-\mu \mathrm{m}$ thickness to observe non-stained histologic specimens under a fluorescence microscope and finally embedded in paraffin. Their images were captured using Biozero BZ-8000 (KEYENCE Co, Ltd, Osaka, Japan). In 3 other groups, the lungs were fixed with the fixing solution $(10 \%$ phosphatebuffered formalin solution: OCT compound $=4: 1$ ) at the same pressure and embedded in paraffin. In all groups, paraffin-embedded sections were cut at $4-\mu \mathrm{m}$ thickness and stained with hematoxylin-eosin and Masson trichrome. Light microscope images from histologic specimens were captured using an Olympus BX51-FL (Olympus Co, Ltd, Tokyo, Japan). Five fields at $40 \times$ magnification of the left lung of each animal were observed under a light microscope. The score of each field was allotted, and the mean score was calculated. Morphologic changes in fibrotic lungs were quantified according to a numeric scale from 0 to 8 as described by Ashcroft and colleagues. ${ }^{16}$ Briefly, the score was as follows: 0, normal; 1, minimal fibrous thickening; 2 , minimal to moderate; 3 , moderate; 4 , 
moderate or more; 5 , increased fibrosis with definite damage; 6 , moderate structural distortion; 7 , severe distortion of structure and large fibrous areas; and 8 , total fibrous obliteration.

\section{Immunohistochemistry}

To examine the relationship between cell differentiation and morphologic changes of implanted grafts, we performed immunohistochemical staining with anti-thyroid transcription factor-1 (TTF-1) and anti-Clara cell secretory protein (CCSP) antibody. Immunohistochemistry for TTF-1 and CCSP was performed as follows. After deparaffinization, sections were incubated in $10 \mathrm{mmol} / \mathrm{L}$ citric buffer $(\mathrm{pH} 6)$ and heated for 10 minutes. After nonspecific sites were blocked with phosphate-buffered saline containing 5\% milk and $0.3 \%$ Triton X-100 (Wako Pure Chemical Industries, Osaka, Japan), sections were incubated for 1 hour at room temperature with primary antibodies. The primary antibodies used were as follows: anti-TTF-1 mouse monoclonal antibody (1:50 dilution; clone 8G7G3/1, Thermo Fisher Scientific, Fremont, Calif) and anti-CCSP rabbit polyclonal antibody (1:50 dilution; SCGB1A1, LifeSpan BioSciences, Seattle, Wash). After thorough washing in phosphate-buffered saline containing $0.1 \%$ Nonidet P-40 (Nacalai Tesque Inc, Kyoto, Japan), sections were incubated with appropriate secondary fluorescence-labeled antibodies for 30 minutes at room temperature and stained with DAPI (Dojindo Laboratories, Kumamoto, Japan). Secondary antibodies used were as follows: ImmPRESS anti-mouse Ig (1:500 dilution; Vector Laboratories, Burlingame, Calif) and Cy3 for anti-TTF-1 and ImmPRESS anti-rabbit Ig (1:500 dilution; Vector Laboratories) and Alexa594 for anti-CCSP. Immunostaining fluorescence was observed, and images were captured using a Leica CW4000 system (Leica Microsystems Co, Ltd, Wetzlar, Germany).

\section{Statistical Analysis}

All values are expressed as mean \pm standard deviation. Statistical analyses were performed using the Student $t$ test with SPSS version 11.0.1 (SPSS Inc, Chicago, Ill). In addition, differences in Ashcroft score at different time points were analyzed by 1-way repeated-measure analysis of variance corrected for multiple comparisons by Bonferroni correction comparing all pairs of columns.

\section{RESULTS}

Histologic Changes and Evaluation of Fibrotic Change in the Lung Using the Ashcroft Score after Intratracheal Administration of Saline and Bleomycin Hydrochloride

To evaluate whether our models were feasible as models of pulmonary fibrosis for a long-term period ranging over 16 weeks, we first examined the histologic changes 4,8 , 12 , and 16 weeks after intratracheal instillation of saline and BLM. In the saline group, no histologic changes were recognized at $4,8,12$, and 16 weeks (Figure 1, A-D, $\times 40$ ). On the other hand, in the BLM group, various findings of fibrotic changes, such as in bronchial and alveolar wall thickness, distortion of structure, and fibrous mass, were recognized at each week (hematoxylin-eosin staining and Masson's trichrome staining, $\times 40$ ) (Figure 1, E-L) compared with those in the saline group.
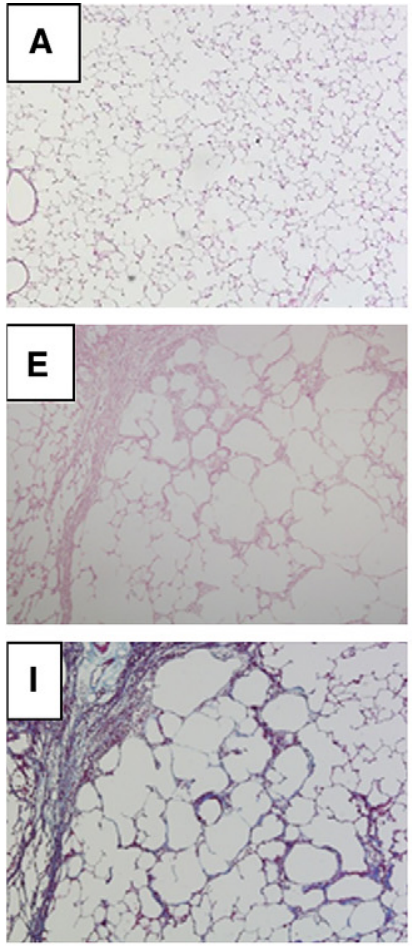

$4 \mathrm{~W}$
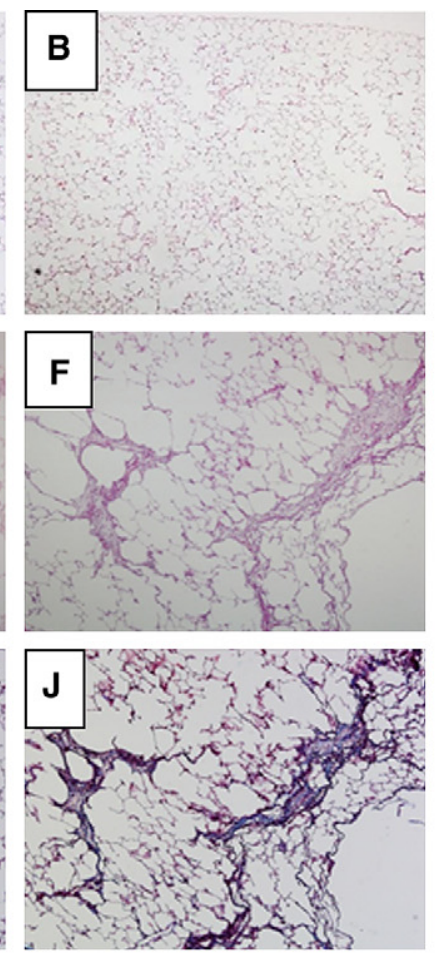

$8 \mathrm{~W}$
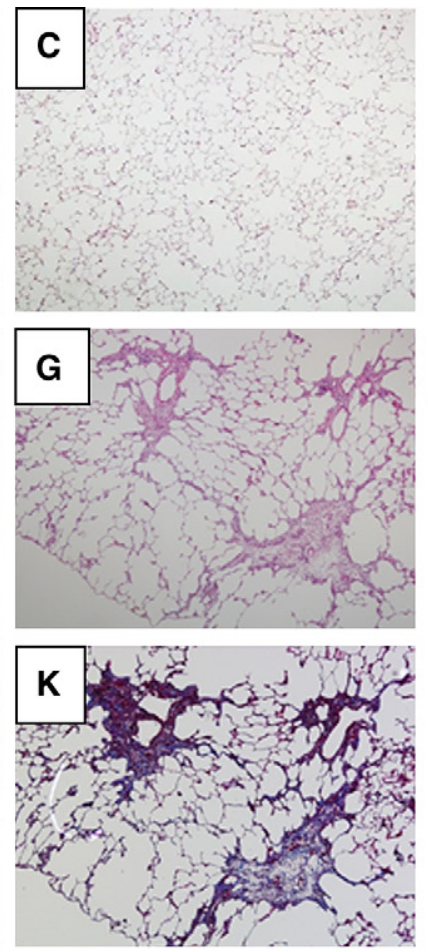

$12 \mathrm{~W}$
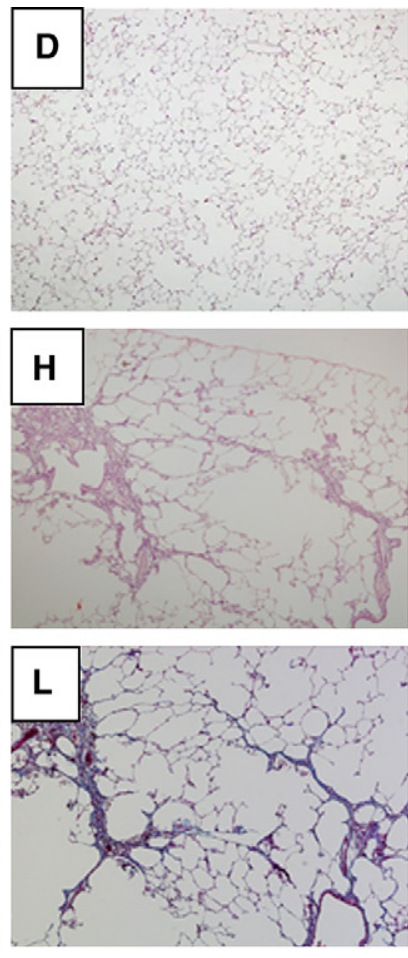

$16 \mathrm{~W}$

FIGURE 1. Histologic examination of BLM-induced pulmonary fibrosis. Rats were intratracheally administered saline or BLM (4.5 mg/kg). At 4, 8,12 , and 16 weeks, rats were killed and histologic examination was performed by hematoxylin-eosin staining (A-H) and Masson's trichrome staining (I-L). All images were taken at the same power (original magnification, $\times 40$ ). A-D, Saline-administered group. E-L, BLM-administered group. Each photograph is representative of 6 animals that were treated in the same way. E-L, Various fibrotic changes were recognized and alveolar structures were destroyed. 

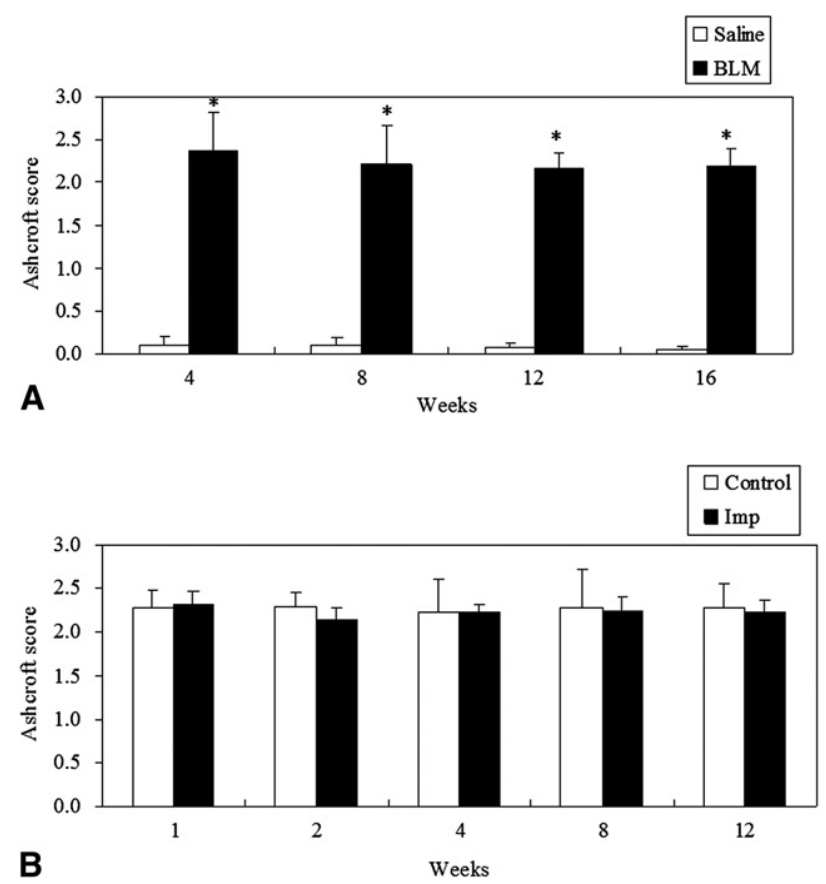

FIGURE 2. Evaluation of fibrotic change in the lung using numeric fibrotic score. Histologic examination in the left lung was performed by hematoxylin-eosin staining. Histologic score was determined by 1 pathologist and 1 surgeon as described in "Materials and Methods." A, Saline and BLM groups. Data are presented as mean \pm standard deviation in each group of 6 animals. $* P<.05$ between saline and BLM groups. B, Control and implantation groups. Data are presented as mean \pm standard deviation in the control group of 5 animals and the implantation group of 4 animals. BLM, Bleomycin; Imp, implantation.

The data of Ashcroft score are shown for both the saline and BLM groups (Figure 2, $A$ ). In the BLM group, Ashcroft scores at each of $4,8,12$, and 16 weeks were similar, namely, between 2.0 and 2.5, and there was no significant difference in mean score. Pulmonary fibrosis was already completed 4 weeks after BLM administration, and spontaneous remission did not occur for a long-term period ranging over 16 weeks. In the saline group, fibrotic change was not recognized at 4, 8, 12, or 16 weeks. At each week, Ashcroft scores in the BLM group were significantly higher than those in the saline group $(P<.01)$.

\section{Evaluation of Fibrotic Change in the Lung of Control and Implantation Groups Using the Ashcroft Score}

In the control and implantation groups, various findings of fibrotic changes like those in the BLM group were also recognized in each week (not shown). Ashcroft score data are shown for both control and implantation groups (Figure 2, B). In the 2 groups, Ashcroft scores in recipient lung field were similar, namely, between 2.0 and 2.5, and there was no significant difference in mean scores between each week. In addition, within each week, there was no significant difference between the mean scores for both groups. There was no therapeutic antifibrotic effect of CyA administered to prevent immune response to GFP protein.

\section{Morphologic Changes of Implanted Fetal Lung Tissues in Fibrotic Lung}

Figure 3 shows morphologic changes of fetal lung tissues at $1,2,4,8$, and 12 weeks after the implantation into fibrotic lung. Implanted fetal lung tissues could survive in the fibrotic lung. Although immunosuppression was performed to prevent an immunoresponse to GFP protein in fetal lung tissues, a few core necrotic lesions were present. With the use of a fluorescence microscope, the recipient lung could be clearly distinguished because of the luminescence of grafts (Figure 3, $A, B, D, E, G, H, J, K, M, N$ ). The alveolar spaces in implanted fetal lung were narrow at 1 and 2 weeks (Figure 3, $A-F$ ), but expanded a little with the passage of time. The connection between the recipient lungs and fetal lungs was recognized morphologically, particularly in the alveolar area (Figure 3, G-O). The epithelial cells in implanted fetal lungs changed from a pseudoglandular shape to thinner and type I-like pneumocytes with the passage of time.

\section{Thyroid Transcription Factor-1 and Clara Cell Secretory Protein Expression of the Graft With the Passage of Time}

One week after implantation, most epithelial cells appeared to be cuboidal in shape, and the structures such as the bud of terminal bronchiole and alveolar duct were also recognized to some extent in the graft. TTF-1 was well stained in the cuboidal cells. On the other hand, CCSP was stained in the bronchiolar epithelial cells of the bud of terminal bronchiole but was not stained in the cuboidal cells (Figure 4, A). After 4 weeks, the implanted fetal tissues further differentiated. Cuboidal cells decreased, and bronchiole and alveolar duct mostly accounted for them. The alveoli were partly covered by type I pneumocytelike cells. Both TTF-1 and CCSP were stained in the bronchiolar epithelial cells of the bronchiole and the alveolar duct. Some of the alveolar cells in implanted lung were slightly stained by TTF-1 but not by CCSP (Figure $4, B$ ). After 12 weeks, the alveolar spaces were filled with air and implanted fetal tissues were recognized as structures like postnatal lungs. CCSP was stained in the bronchial epithelial cells, but TTF-1 expression was slightly stained in the bronchial cells and alveolar cells (Figure 4, C).

\section{DISCUSSION}

BLM-induced pulmonary fibrosis in rodents has been widely used as a good model exhibiting histologic findings similar to human idiopathic pulmonary fibrosis. ${ }^{4}$ Various experiments, such as the use of many antifibrotic agents, ${ }^{4}$ 
Non-stained
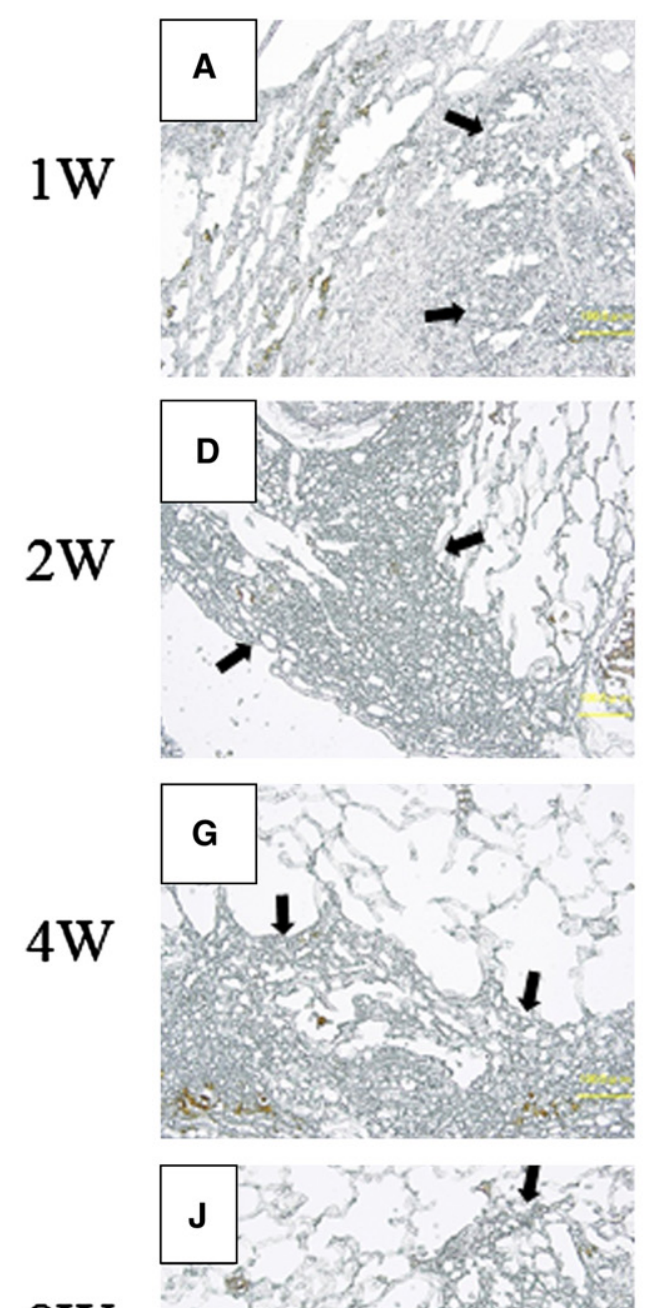

\section{W}
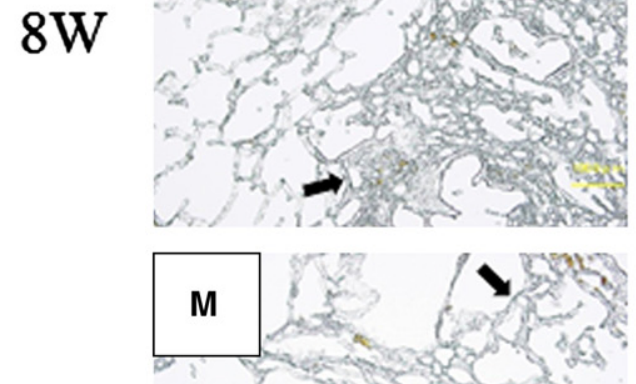

$12 \mathrm{~W}$

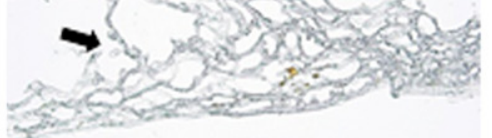

Fluorescence
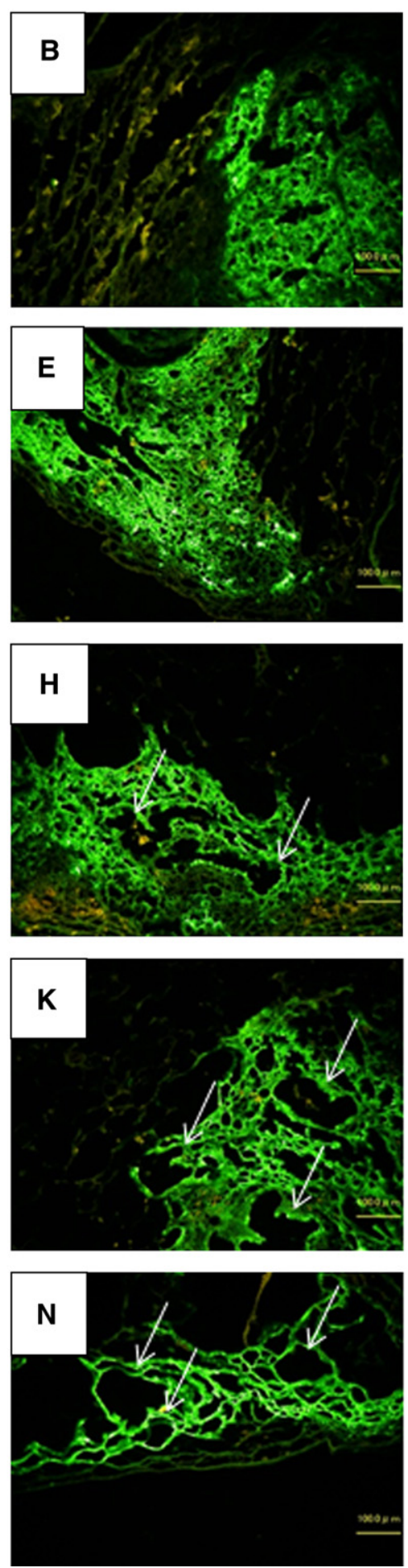

H\&E-stained
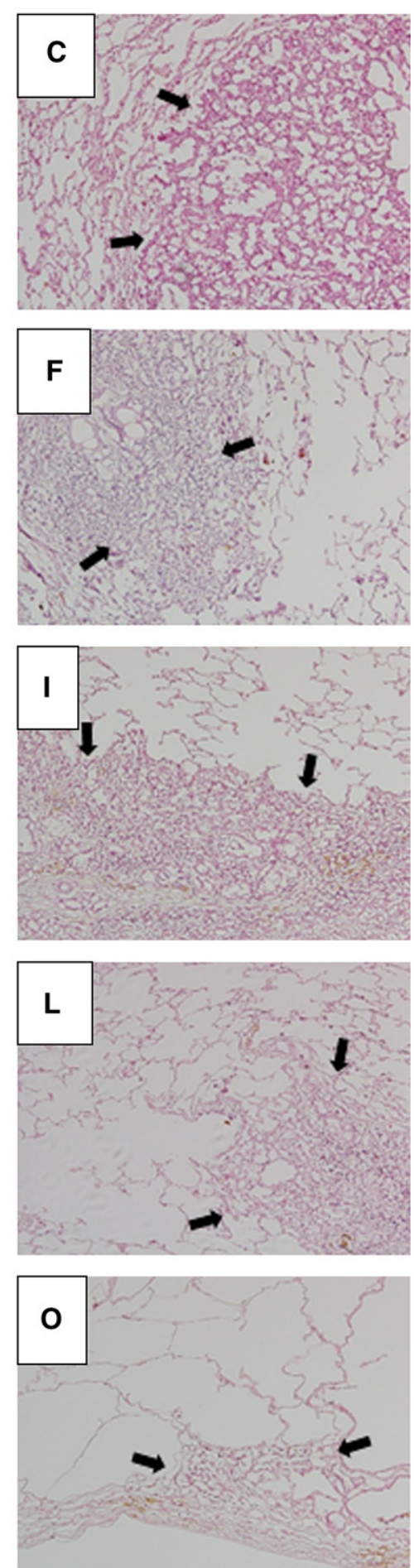

FIGURE 3. Morphologic change of implanted fetal lung tissues in the BLM-induced fibrotic lung. Fetal lung tissues and surrounding area were examined after 1 (A-C), 2 (D-F), 4 (G-I), 8 (J-L), and 12 (M-O) weeks. Each photograph is representative of 4 animals that were treated in the same way. Non-stained specimens were observed by white light (A, D, G, J, M) and fluorescence (B, E, H, K, N) microscopy. Hematoxylin-eosin-stained specimens (C, F, I, L, O) are shown at $100 \times$ magnification. Arrows show the border zones between injected and native tissues. Thin arrows show the widened alveolar spaces in $\mathrm{H}$, $\mathrm{K}$, and N. Bar $=100.0 \mu \mathrm{m} . H \& E$, Hematoxylin-eosin. 


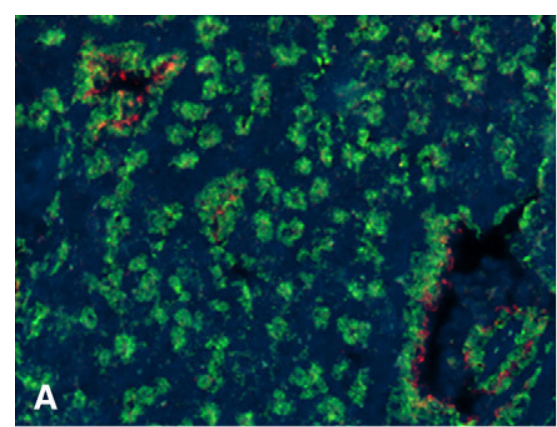

$1 \mathrm{~W}$

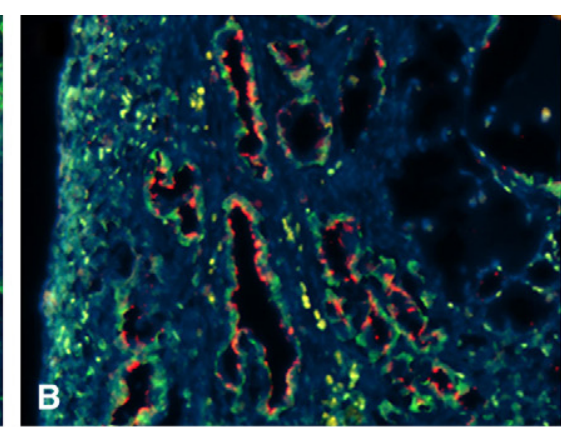

$4 \mathrm{~W}$

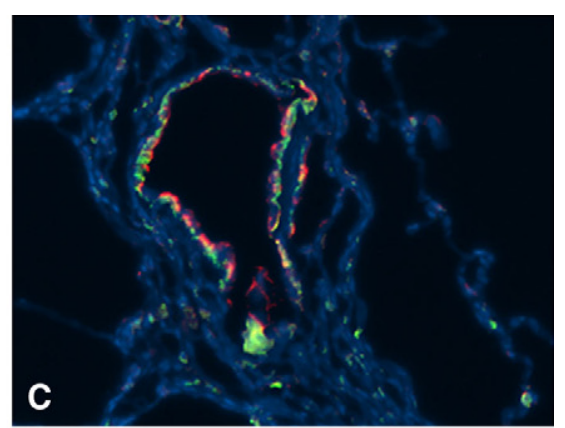

$12 \mathrm{~W}$

FIGURE 4. Expression of TTF-1 and CCSP in implanted fetal lung tissues. Adjacent serial sections were stained for TTF-1 (Cy3; green fluorescence) or CCSP (Alexa594; red fluorescence) with DAPI (blue fluorescence) counterstain at 1 (A), 4 (B), and 12 (C) weeks after implantation. A-C, 100× magnification.

intratracheal transplantation of alveolar type II cells, ${ }^{9}$ and administration of bone marrow-derived stem cells, ${ }^{6}$ have been tried to prevent fibrotic progression in the BLM model. Chaudhary and colleagues ${ }^{17}$ indicated that the switch between inflammatory phase and fibrotic phase appears to occur at approximately day 9 in the rat BLM model. In addition, continuous fibrotic changes result in deposition of matrix and distortion of lung structure for as long as 28 or 35 days. In this study, we observed our fibrotic lung model up to 36 weeks and demonstrated that fibrotic changes continued over a long period of time with a single administration of BLM by our method. The Ashcroft score was $2.14 \pm 0.23(n=6)$ at 36 weeks and showed no significant difference compared with those at $4,8,12$, and 16 weeks. In our model, once fibrotic changes were completed, spontaneous remission was not recognized. Accordingly, this BLM model in rats is useful for observation over an extended period of time. In this study, CyA was administered to prevent an immune response to GFP protein in the control and implantation groups. However, the Ashcroft score in the BLM group was almost the same as those in the control and implantation groups. CyA, which is one of the great variety of compounds used in experimental attempts to show an antifibrotic effect in the BLM model, ${ }^{18}$ is a drug tested in clinical trials for idiopathic pulmonary fibrosis treatment. ${ }^{19}$ However, there was no antifibrotic effect of the administration of CyA in this study. We postulated that the reasons for this were that the administered dose of CyA was extremely small and the time of initiation of administration of CyA was 4 weeks after BLM administration, corresponding to the fibrotic phase in this study. In general, antifibrotic agents are administered in the anti-inflammatory phase.

The current study demonstrated that fetal lung tissues could also survive, grow further, and differentiate in the BLM-induced fibrotic lungs, as we previously reported the same phenomenon in normal adult lungs. ${ }^{11}$ In this previous study, we used inbred animals as the donors and recipients to avoid immune responses. However, in this study, we used fetal lung tissues of GFP rats as the donor tissues. As a result, we could more clearly distinguish the border zones between injected and native tissues. Although it was consequently necessary to use CyA as an immunosuppressive agent for GFP protein, its dose was small and there was no effect on the engraftment.

At 1 to 2 weeks after implantation, the morphology of the grafts was similar to that in the pseudoglandular stage of fetal rat lung and epithelial cells were mainly composed of cuboidal cells. However, at 4 and 8 to 12 weeks, alveolar units were formed in some lesions and epithelial cells were mainly composed of type I pneumocyte-like cells. These phenomena were similar to changes over time as described in a previous report. ${ }^{11}$ However, air spaces of grafts implanted in fibrotic lungs were clearly narrow compared with those in normal lungs, although they expanded slightly at 4,8 , and 12 weeks. One cause of this might be low compliance of a recipient's lung because of BLM-induced fibrotic lung. Moreover, these results might be partly due to less mechanical forces in the recipient lungs. We previously demonstrated that mechanical forces by partial lung resection might facilitate proliferation and maturation of implanted fetal tissues. ${ }^{11}$ In this study, partial removal of the left lung was only performed as closure of an inlet for implantation on a recipient's lung to prevent air leakage without removal of the postcaval lobe of the recipient's lung. Although air spaces were narrow owing to low compliance of the lung and less mechanical forces, survival and differentiation of grafts in the fibrotic lungs might be considered one important aspect of this study.

TTF-1 is a member of the Nkx2 family of homeodomaincontaining proteins expressed in forebrain, thyroid, and lung. In the lung, TTF-1 plays a critical role in the regulation of lung morphogenesis, epithelial cell differentiation, and expression of genes on which perinatal respiratory adaptation depends. ${ }^{20,21}$ TTF-1 is expressed in respiratory epithelial cells at all stages throughout lung morphogenesis. In addition, in the postnatal lung, TTF-1 is also expressed primarily in alveolar type II epithelial cells. ${ }^{22}$ On the other hand, Clara cells have been shown to be the progenitors of 
ciliated cells in the bronchioles and to be critical for epithelial renewal after progenitor cell depletion. ${ }^{21,23}$ CCSP is expressed in Clara cells and regulated by TTF- $1 .{ }^{24}$ In this study, TTF-1 was well stained in most cuboidal cells and epithelial cells of terminal bronchiole in the graft at 1 week. Later, the TTF-1 expression was mainly recognized in bronchial epithelial cells but was also recognized in alveolar epithelial cells through the passage of time from 4 to 12 weeks. In addition, the CCSP expression was recognized in only bronchial epithelial cells. On the basis of the TTF-1 and CCSP expression data, these results resemble the pattern in the process of normal lung morphogenesis. Therefore, in recipient lungs, the graft may pass through the normal fetal rat lung morphogenetic course.

Fetal rat lung tissue has (1) great potential for further growth, (2) excellent potential for differentiation and proliferation, and (3) mesenchymal tissue as a suitable scaffold. ${ }^{12}$ The results of this study might be dependent on these characteristics of fetal lung tissue. However, this study demonstrated the survival and differentiation of implanted fetal lung tissues in BLM-induced completed fibrotic lung. In previous studies, the use of many anti-inflammatory drugs ${ }^{4}$ and the administration of bone marrow-derived stem cells, ${ }^{6}$ alveolar type II cells, ${ }^{9}$ and others were methods to prevent the fibrotic process. Our study can be considered to show another new regenerative approach using fetal rat lung tissue for already completely fibrotic lung. Furthermore, we could demonstrate that implanted fetal lung tissues might follow the normal fetal rat lung morphogenetic course in recipient lung. This phenomenon shows a part of the mechanism of differentiation of implanted fetal lung tissues. However, further research on the morphogenetic mechanism is needed. This could lead to the development of appropriate techniques to induce implanted fetal lung tissue to become mature lung and increase the donors freely. On the other hand, we are left with the issues of whether implantation of fetal lung tissue can improve respiratory function in fibrotic lung model and which cells of the fetal rat lung tissue can play key roles in the survival and differentiation. In addition, our experimental model should be applied to large animals and allografts. Furthermore, ethical concern might remain about the use of fetal lung tissue to treat adult human diseases. Nevertheless, we believe that fetal lung tissue is a potentially useful option for investigating lung regeneration.

\section{CONCLUSIONS}

Fetal rat lung fragments survived and differentiated in BLM-induced completely fibrotic lung.
The authors thank Eiji Kobayashi, Division of Development of Advanced Treatment, Center for Development of Advanced Medical Technology, Jichi Medical University, for providing GFP Lewis rats.

\section{References}

1. American Thoracic Society. Idiopathic pulmonary fibrosis: diagnosis and treatment. International consensus statement. Am J Crit Care Med. 2000;161:646-64.

2. Gross TJ, Hunninghake GW. Idiopathic pulmonary fibrosis. N Engl J Med. 2001; 345:517-25

3. Kim DS, Collard HR, King TE Jr. Classification and natural history of the idiopathic interstitial pneumonias. Proc Am Thorac Soc. 2006;3:285-92.

4. Moeller A, Ask K, Warburton D, Gauldie J, Kolb M. The bleomycin animal model: a useful tool to investigate treatment options for idiopathic pulmonary fibrosis? Int J Biochem Cell Biol. 2008;40:362-82.

5. Roomans GM. Tissue engineering and the use of stem/progenitor cells for airway epithelium repair. Eur Cell Mater. 2010;19:281-99.

6. Rojas M, Xu J, Woods CR, Mora AL, Spears W, Roman J, et al. Bone marrowderived mesenchymal stem cells in repair of the injured lung. Am J Respir Cell Mol Biol. 2005;33:145-52.

7. Hashimoto N, Jin H, Liu T, Chensue SW, Phan SH. Bone marrow-derived progenitor cells in pulmonary fibrosis. J Clin Invest. 2004;113:243-52.

8. Kotton DN, Fabian AJ, Mulligan RC. Failure of bone marrow to reconstitute lung epithelium. Am J Respir Cell Mol Biol. 2005;33:328-34.

9. Serrano-Mollar A, Nacher M, Gay-Jordi G, Xaubet A, Bulbena O. Intratracheal transplantation of alveolar type II cells reverses bleomycin-induced lung fibrosis. Am J Respir Crit Care Med. 2007;176:1261-8.

10. Nichols JE, Cortiella J. Engineering of a complex organ. Progress toward development of a tissue-engineered lung. Proc Am Thorac Soc. 2008;5:723-30.

11. Kenzaki K, Sakiyama S, Kondo K, Yoshida M, Kawakami Y, Takehisa M, et al Lung regeneration: implantation of fetal rat lung fragments into adult lung parenchyma. J Thorac Cardiovasc Surg. 2006;131:1148-53.

12. Copland I, Post M. Lung development and fetal lung growth. Paediatr Respir Rev. 2004;5(Suppl):S259-64.

13. Hakamata Y, Tahara K, Uchida H, Sakuma Y, Nakamura M, Kume A, et al. Green fluorescent protein-transgenic rat: a tool for organ transplantation research. Biochem Biophys Res Commun. 2001;286:779-85

14. Goto H, Senba T, Sato M, Minami T. Intratracheal administration of bleomycin via a catheter in unanesthetized rats. Exp Anim. 2004;53:113-9.

15. Liu M, Xu J, Tanswell AK, Post M. Stretch-induced growth-promoting activities stimulate fetal rat lung epithelial cell proliferation. Exp Lung Res. 1993;19: 505-17.

16. Ashcroft T, Simpson JM, Timbrell V. Simple method of estimating severity of pulmonary fibrosis on a numerical score. J Clin Pathol. 1998;41:467-70.

17. Chaudhary NI, Schnapp A, Park JE. Pharmacologic differentiation of inflammation and fibrosis in the rat bleomycin model. Am J Respir Crit Care Med. 2006; 173:769-76.

18. Moolman JA, Bardin PG, Rossouw DJ, Joubert JR. Cyclosporin as a treatment for interstitial lung disease of unknown aetiology. Thorax. 1991;46:592-5.

19. Alton EW, Johnson M, Turner-Warwick M. Advanced cryptogenic fibrosing alveolitis: preliminary report on treatment with cyclosporine A. Respir Med. 1989; 83:277-9.

20. Maeda Y, Dave V, Whitsett JA. Transcriptional control of lung morphogenesis Physiol Rev. 2007;87:219-44.

21. Minoo P, Hamdan H, Bu D, Warburton D, Stepanik P, deLemos R. TTF-1 regulates lung epithelial morphogenesis. Dev Biol. 1995;172:694-8.

22. Kelly SE, Bachurski CJ, Baurhans MS, Glasser SW. Transcription of the lung specific surfactant protein $\mathrm{C}$ gene in mediated by thyroid transcription factor 1 . J Biol Chem. 1996;271:6881-8.

23. Bishop AE. Pulmonary epithelial stem cells. Cell Prolif. 2004;37:89-96.

24. Bohinski RJ, DiLauro R, Whitsett JA. The lung-specific surfactant protein B gene promoter is a target for thyroid transcription factor 1 and hepatocyte nuclear factor 3 , indicating common factors for organ-specific gene expression along the foregut axis. Mol Cell Biol. 1994;14:5671-81. 